\title{
29. Enhancement Tumor Immunity-Modification of Autochthonous Antigen with Mercaptounadecahydroduodecaborate
}

\author{
Hiroshi Hatanaka, Hisashi Yamada and Keiji Sano \\ Department of Neurosurgery, University of Tokyo
}

This is the 2nd publication following the paper given at the 58th Annual Meeting of the Society of Neurological Surgeons held in April, 1967 at Portland, Oregon.

The possibility of tumor-specific antigens has been recognized by many people by now. But many trials of application of such antigens to therapeutic purpose have so far failed probably owing to very low antigenicity of the antigen itself. Hatanaka invented the preparation of tumor antigens modified with one of the boron-sulfhydryl alkylating agents, $\mathrm{Na}_{2} \mathrm{~B}_{12} \mathrm{H}_{11} \mathrm{SH}$, which forms a strong bond with tumor proteins, to enhance the antigenicity of the tumor antigens.

The material was an isogeneic brain tumor originally induced by methylcholanthrene and transplanted subcutaeously into $\mathrm{C} 3 \mathrm{H} / \mathrm{HeJ}$ mice. The mice that had received the vaccine that had been made by incubating the tumor homogenate with an isotonic solution of the compound demonstrated a marked tumor growth retardation in comparison to the non-treated group of mice. And further, the vaccine showed a statistically significant tumor growth suppression even when it was given after the tumor was transplanted.

It was clarified that this tumor-suppressive effect did not owe to some antitumor action of the compound, by a fact that the administration of the compound alone had not suppressed the tumor growth and by an antigen-clearance test.

\section{Hyperbaric Oxygen Therapy as a Postoperative Management in Neurosurgery}

\author{
Kenzo Matsuoka, Tohru Uozumi, Mitsumasa Kano, Tsukasa Kishida \\ and Kazuyasu NAKAO \\ 1st Dept. of Surgery, Osaka Univ. Medical School
}

Recently, it is reported that hyperbaric oxygen therapy has a possible value in some diseases in which hypoxia is present. However, it has not been clarified whether this therapy is effective for the unconsciousness in the postoperative course of neurosurgery. 